\title{
Entanglement of Assistance
}

\author{
David P. DiVincenzo ${ }^{1}$, Christopher A. Fuchs ${ }^{2}$, Hideo Mabuchi ${ }^{2}$, John A. \\ Smolin ${ }^{1}$, Ashish Thapliyal ${ }^{3}$, and Armin Uhlmann ${ }^{4}$ \\ 1 IBM Research Division, Yorktown Heights, NY 10598, U. S. A. \\ divince/smolin@watson.ibm.com \\ 2 Bridge Laboratory of Physics 12-33, California Institute of Technology, Pasadena, \\ CA 91125, U. S. A. cfuchs/hmabuchi@cco.caltech.edu \\ 3 Department of Physics, University of California at Santa Barbara, Santa Barbara, \\ CA 93106, U. S. A. ash@physics.ucsb.edu \\ 4 Institut für Theoretische Physik, Universität Leipzig, Augustusplatz 10/11, \\ D-04109 Leipzig, Germany. Armin.Uhlmann@itp.uni-leipzig.de
}

\begin{abstract}
The newfound importance of "entanglement as a resource" in quantum computation and quantum communication behooves us to quantify it in as many distinct ways as possible. Here we explore a new quantification of entanglement of a general (mixed) quantum state for a bipartite system, which we name entanglement of assistance. We show it to be the maximum of the average entanglement over all ensembles consistent with the density matrix describing the bipartite state. With the help of lower and upper bounds we calculate entanglement of assistance for a few cases and use these results to show the surprising property of superadditivity. We believe that this may throw some light on the question of additivity of entanglement of formation.
\end{abstract}

\section{Introduction}

Much of the preoccupation of classical information theory is in making the correlation between two ends of a communication channel - that is, between a sender and a receiver - as high as possible. This is what (classical) communication is about. In contrast, this is only part of the story for the fledgling field of quantum information theory. The quantum world brings with it a new resource that senders and receivers can share: quantum entanglement, the stuff Einstein-Podolsky-Rosen pairs and Bell-inequality violations are made of. This new resource, of all the things in quantum information theory, is the most truly "quantum" of the lot. It is a resource because of the myriad ways in which it is starting to be used. The list of its applications already includes quantum key distribution [1], quantum-state teleportation [2], entanglement-enhanced classical communication [3], error-correction for quantum computers [4], entanglementassisted multi-party communication [5], and better control of atomic frequency standards [6] among other things. The list grows every day.

The newfound importance of "entanglement as a resource" behooves us to quantify entanglement in as many distinct ways as possible. To this end, we 
explore a new quantification of the entanglement of a general (mixed) quantum state for a bipartite system. This quantity we dub the entanglement of assistance. It is something of a dual notion to that of the entanglement of formation studied by Bennett, DiVincenzo, Smolin and Wootters [7]. It can be motivated in the following way.

Consider three players Alice, Bob, and Charlie, who jointly possess many copies of a tripartite quantum system each described by the pure state $\left|\Psi^{\mathrm{ABC}}\right\rangle$. It follows that Alice and Bob, considered in isolation, possess many copies of the (generally mixed) state $\rho^{\mathrm{AB}}=\operatorname{tr}_{\mathrm{C}}\left|\Psi^{\mathrm{ABC}}\right\rangle\left\langle\Psi^{\mathrm{ABC}}\right|$. Suppose now that Alice and Bob need to use their shared quantum system for one of the tasks above, teleportation for instance. However, very unfortunately, Charlie is not immediately available to pass his systems over for their use. Since their state may not be very pure or entangled for that matter, they may be seriously impaired by this. How might Charlie help in spite of his constraint?

One thing he can do is perform a measurement on each system that he possesses so that Alice and Bob will have pure states conditioned on his outcomes. If he transmits the classical information so obtained, they may be able to use it to their benefit. For instance, if several of his measurements reveal that Alice and Bob actually possess the pure state $\left|\Psi^{\mathrm{AB}}\right\rangle$, then by the result of Bennett, et al. [8], they can convert these quantum states into maximally-entangled singlet states at a rate of $S\left(\rho^{\mathrm{A}}\right)=-\operatorname{tr}\left(\rho^{\mathrm{A}} \log _{2} \rho^{\mathrm{A}}\right)$, where $\rho^{\mathrm{A}}=\operatorname{tr}_{\mathrm{B}}\left|\Psi^{\mathrm{AB}}\right\rangle\left\langle\Psi^{\mathrm{AB}}\right|$. In general then, for a measurement that creates the states $\left|\Psi_{i}^{\mathrm{AB}}\right\rangle$ with probabilities $p_{i}$, i.e., an ensemble $\mathcal{E}=\left\{p_{i},\left|\Psi_{i}^{\mathrm{AB}}\right\rangle\left\langle\Psi_{i}^{\mathrm{AB}}\right|\right\}$, the average rate for conversion to singlets will be

$$
E(\mathcal{E})=\sum_{i} p_{i} S\left(\rho_{i}^{\mathrm{A}}\right)=-\sum_{i} p_{i} \operatorname{tr}\left(\rho_{i}^{\mathrm{A}} \log _{2} \rho_{i}^{\mathrm{A}}\right) .
$$

Since Charlie is a friend of Alice and Bob, he should choose his measurement so that this average rate is maximized. By a theorem of Hughston, Jozsa, and Wootters [9] this maximization can be taken over all possible ensembles $\mathcal{E}$ consistent with $\rho^{\mathrm{AB}}$ in the sense that

$$
\rho^{\mathrm{AB}}=\sum_{i} p_{i}\left|\Psi_{i}^{\mathrm{AB}}\right\rangle\left\langle\Psi_{i}^{\mathrm{AB}}\right| .
$$

Therefore the resulting maximal rate is a number intrinsic to $\rho^{\mathrm{AB}}$, depending upon no further details of the state $\left|\Psi^{\mathrm{ABC}}\right\rangle$. This is the entanglement of assistance $A\left(\rho^{\mathrm{AB}}\right)$ for $\rho^{\mathrm{AB}}$ :

$$
A\left(\rho^{\mathrm{AB}}\right)=\max _{\mathcal{E}} E(\mathcal{E}) .
$$

This quantity seems to be dual to the entanglement of formation, which is defined by

$$
F\left(\rho^{\mathrm{AB}}\right)=\min _{\mathcal{E}} E(\mathcal{E}) .
$$

In this paper, we demonstrate several properties possessed by entanglement of assistance, calculating it explicitly for some specialized cases. We exhibit a general upper bound, a particular upper bound specialized to the two-qubit case and a lower bound for diagonal density matrices, on the entanglement of 
asistance. With the aid of the last two, we prove a fairly surprising property: the entanglement of assistance is in some cases superadditive. That is, there exist density operators $\rho_{1,2}^{\mathrm{AB}}$ for which

$$
A\left(\rho_{1}^{\mathrm{AB}} \otimes \rho_{2}^{\mathrm{AB}}\right)>A\left(\rho_{1}^{\mathrm{AB}}\right)+A\left(\rho_{2}^{\mathrm{AB}}\right) .
$$

This means that if Charlie performs entangled measurements on his separate copies of the system, he can be more effective in helping Alice and Bob than he would have been otherwise.

\section{Properties and bounds}

The average entanglement of an ensemble is invariant under local unitary transformations and non-increasing under general local operations with classical communication [7,8]; so entanglement of assistance also has these properties. In addition to this it is easy to see that since there is a maximization over all possible pure-state realizations of the density matrix, the entanglement of assistance $A$ is concave. That is,

$$
A\left(\sum_{i} p_{i} \rho_{i}\right) \geq \sum_{i} p_{i} A\left(\rho_{i}\right)
$$

We note here that the entanglement of assistance is the least of all concave functions coinciding on pure states with their partial entropy i.e. entropy as seen from one side, while entanglement of fomation is the greatest convex function with that property. This may be seen as a sign of the mentioned duality between the two.

Since we do not have a general formula 2 for entanglement of assistance of a given density matrix, upper and lower bounds are of great importance. We have found a few bounds which have helped us calculate the entanglement of assistance in some cases and have been of immense value in proving its superadditivity.

\subsection{Upper bounds}

We have found an upper bound - the entropic bound - which works in general, and another upper bound - the fidelity bound - which works for the simplest case of one qubit on Alice's side and one on Bob's side.

\footnotetext{
${ }^{1}$ Local operations are those that Alice and Bob can perform on their own part of the system with possible synchronization using classical messages. However, they may not send each other quantum systems nor can they come together and perform a joint quantum operation on the system.

${ }^{2}$ We will present a formula for a non-trivial class of rank two density matrices in a forthcomming publication.
} 
Entropic bound: The entanglement of assistance is never greater than the minimum of the partial entropy as seen by Alice or Bob:

$$
A(\rho) \leq \operatorname{Min}\left[S\left(\operatorname{tr}_{\mathrm{A}} \rho\right), S\left(\operatorname{tr}_{\mathrm{B}} \rho\right)\right] .
$$

To prove this assume that $A(\rho)$ is achieved by the pure-state ensemble $\mathcal{E}=$ $\left\{p_{i}, \Pi_{i}=\left|\psi_{i}\right\rangle\left\langle\psi_{i}\right|\right\}$, then by the concavity of Von Neumann entropy $S$ we have,

$$
S\left(\operatorname{tr}_{\mathrm{A}} \rho\right) \geq \sum_{i} p_{i} S\left(\operatorname{tr}_{\mathrm{A}} \Pi_{i}\right)=A(\rho)
$$

The same inequality holds with trace over A replaced by trace over B. Combining them we get the entropic bound.

Since the Von Neumann entropy is additive and concave the bound is also additive and concave. Thus for states which saturate this bound the entanglement of assistance must be additive. We can also see from the results of section (3) that the entropic bound is zero if and only if the entanglement of assistance is zero. The bound obviously agrees with entanglement of assistance for pure states. It is

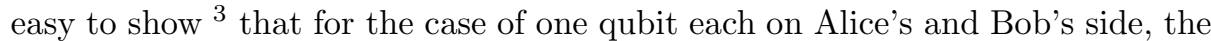
entropic bound is maximum (1 ebit) if and only if the entanglement of assistance is also maximum.

Fidelity bound: For the case of one qubit each with Alice and Bob, we can get a bound that is sometimes stronger than the entropic bound. It says that the entanglement of assistance cannot be greater than the fidelity of the density matrix relative to its complex conjugate in the magic basis $\mathrm{f}^{\mathrm{f}}$ i.e.,

$$
A(\rho) \leq F(\rho, \tilde{\rho})
$$

where the ${ }^{\sim}$ - Hill-Wootters tilde - represents complex conjugation in the magic basis [10] and $F(\rho, \sigma)=\operatorname{tr} \sqrt{\rho^{1 / 2} \sigma \rho^{1 / 2}}$ is the fidelity [11] that is, the square root of the transition probability [12]. To prove the bound the main thing we need is a classical inequality on the binary Shannon entropy [13]. It is,

$$
\begin{aligned}
H_{2}(x) & =-x \log _{2} x-(1-x) \log _{2}(1-x) \\
& \leq 2 \sqrt{x(1-x)} .
\end{aligned}
$$

Equality is achieved in this if and only if $x=0, \frac{1}{2}, 1$. The other facts we need are that for a pure state $\Pi_{i}$ in a decomposition of $\rho$, the largest eigenvalue, $\lambda_{1}\left(\rho_{i}^{\mathrm{A}}=\operatorname{tr}_{\mathrm{B}} \Pi_{i}\right)$, of its reduced density operator is given in terms of the HillWootters tilde by

$$
\lambda_{1}\left(\rho_{i}^{\mathrm{A}}\right)=\frac{1}{2}\left[1+\sqrt{1-\operatorname{tr}\left(\Pi_{i} \tilde{\Pi}_{i}\right)}\right]
$$

\footnotetext{
${ }^{3}$ We show this in a forthcomming publication.

4 The magic basis is: $\left|e_{1}\right\rangle=\frac{|00\rangle\rangle|11\rangle}{\sqrt{2}} ;\left|e_{2}\right\rangle=\frac{|00\rangle-|11\rangle}{-i \sqrt{2}} ;\left|e_{3}\right\rangle=\frac{|01\rangle+|10\rangle}{-i \sqrt{2}} ;\left|e_{4}\right\rangle=\frac{|01\rangle-|10\rangle}{\sqrt{2}}$.
} 
and, that the fidelity function is doubly concave in its two arguments and

$$
F\left(\Pi_{i}, \tilde{\Pi}_{i}\right)=\sqrt{\operatorname{tr}\left(\Pi_{i} \tilde{\Pi}_{i}\right)}
$$

Putting all this together: for any ensemble $\mathcal{E}=\left\{p_{i}, \Pi_{i}\right\}$ consistent with $\rho$ average entanglement is

$$
\begin{aligned}
E(\mathcal{E}) & =\sum_{i} p_{i} S\left(\rho_{i}^{\mathrm{A}}\right) \\
& =\sum_{i} p_{i} H_{2}\left(\lambda_{1}\left(\rho_{i}^{\mathrm{A}}\right)\right) \\
& \leq 2 \sum_{i} p_{i} \sqrt{\lambda_{1}\left(\rho_{i}^{\mathrm{A}}\right)\left(1-\lambda_{1}\left(\rho_{i}^{\mathrm{A}}\right)\right)} \\
& =\sum_{i} p_{i} \sqrt{\operatorname{tr}\left(\Pi_{i} \tilde{\Pi}_{i}\right)} \\
& =\sum_{i} p_{i} F\left(\Pi_{i}, \tilde{\Pi}_{i}\right) \\
& \leq F(\rho, \tilde{\rho}) .
\end{aligned}
$$

The bound in Eq. (8) follows immediately.

Note the conditions for saturating this bound. In particular, in going from Eq. (14) to (15), we see that this new bound can be achieved only if $\rho$ has a decomposition consisting only of maximally entangled and completely unentangled states. Thus the bound can't be tight generally - consider simply a $\rho$ that is pure but not itself maximally entangled. However, we will see in section (4) that this bound is sometimes better than the entropic bound.

\subsection{Lower bounds}

To get lower bounds we use the fact that the average entanglement of any purestate realization of the density matrix gives us a lower bound on the entanglement of assistance. Using this idea we can come up with lower bounds by making systematic pure-state decompositions of the density matrix. For example, average entanglement of the eigenvector decomposition of the density matrix is a lower bound.

Applying the above idea to density matrices diagonal in a product basis we get a lower bound which we name diagonal lower bound. Consider the case of only one qubit on either side so that the product basis can be written as $\{|00\rangle,|01\rangle,|10\rangle,|11\rangle\}$. The density matrix in this product basis looks like,

$$
\rho=\left(\begin{array}{cccc}
\alpha_{1} & 0 & 0 & 0 \\
0 & \alpha_{2} & 0 & 0 \\
0 & 0 & \alpha_{3} & 0 \\
0 & 0 & 0 & \alpha_{4}
\end{array}\right)
$$


and the bound is,

$$
A(\rho) \geq\left(\alpha_{1}+\alpha_{4}\right) H_{2}\left(\frac{\alpha_{1}}{\alpha_{1}+\alpha_{4}}\right)+\left(\alpha_{2}+\alpha_{3}\right) H_{2}\left(\frac{\alpha_{2}}{\alpha_{2}+\alpha_{3}}\right) .
$$

This follows from the pure state realization of $\rho: \mathcal{E}=\left\{p_{i},\left|\psi_{i}\right\rangle \mid i=1, \ldots, 4\right\}$, with

$$
\begin{aligned}
& \left\{p_{i}\right\}=\left\{\left(\alpha_{1}+\alpha_{4}\right) / 2,\left(\alpha_{1}+\alpha_{4}\right) / 2,\left(\alpha_{2}+\alpha_{3}\right) / 2,\left(\alpha_{2}+\alpha_{3}\right) / 2\right\} \\
& \left|\psi_{1}\right\rangle=\left(\sqrt{\alpha_{1} /\left(\alpha_{1}+\alpha_{4}\right)}, 0,0, \sqrt{\alpha_{4} /\left(\alpha_{1}+\alpha_{4}\right)}\right) \\
& \left|\psi_{2}\right\rangle=\left(\sqrt{\alpha_{1} /\left(\alpha_{1}+\alpha_{4}\right)}, 0,0,-\sqrt{\alpha_{4} /\left(\alpha_{1}+\alpha_{4}\right)}\right) \\
& \left|\psi_{3}\right\rangle=\left(0, \sqrt{\alpha_{2} /\left(\alpha_{2}+\alpha_{3}\right)}, \sqrt{\alpha_{3} /\left(\alpha_{2}+\alpha_{3}\right)}, 0\right) \\
& \left|\psi_{4}\right\rangle=\left(0, \sqrt{\alpha_{2} /\left(\alpha_{2}+\alpha_{3}\right)},-\sqrt{\alpha_{3} /\left(\alpha_{2}+\alpha_{3}\right)}, 0\right) .
\end{aligned}
$$

Numerical results using optimization algorithms suggest that this is indeed the exact formula for entanglement of assistance for these density matrices. Also when this result saturates any of the upper bounds we know that it is the answer.

The bound can easily be generalized to higher dimensions 5 . Another thing to note here is that off-diagonal terms between $\{|00\rangle,|11\rangle\}$ and $\{|01\rangle,|10\rangle\}$ don't change the lower bound because we can still use a similar pure state decomposition as before, with slightly modified probabilities.

\section{Examples}

Let us start by characterizing the states that have zero entanglement of assistance. We find that there is a very simple characterization of these states as, states whose density matrix is pure on at least one side. That is, either $\rho=\left|\psi_{A}\right\rangle\left\langle\psi_{A}\right| \otimes \rho_{B}$ or $\rho=\rho_{A} \otimes\left|\psi_{B}\right\rangle\left\langle\psi_{B}\right|$.

To prove this result first note that since the entropic (upper) bound (6i) is zero for such states, these states have zero entanglement of assistance. Let us now prove that only such states can have zero entanglement of assistance. Since entanglement of assistance is zero, every valid pure-state decomposition of $\rho$ must consist exclusively of product states. (For otherwise the average entanglement of that ensemble will be necessarily greater then zero.) So let us focus on an eigendecomposition of $\rho$ : this is an ensemble $\mathcal{E}=\left\{\lambda_{i},\left|\alpha_{i}\right\rangle\left|\beta_{i}\right\rangle\right\}$. (The $\lambda_{i} \neq 0$ are the probabilities for the associated states.)

Now, by the Hughston-Jozsa-Wootters theorem [9], any "unitary reshuffling" of this ensemble is also a valid ensemble. Therefore let us consider a new ensemble $\mathcal{E}^{\prime}=\left\{p_{i},\left|\psi_{i}\right\rangle\right\}$ that is exactly the same as the old one, except in the first two elements. Namely,

$$
\begin{aligned}
& \sqrt{p_{1}}\left|\psi_{1}\right\rangle=\cos \theta \sqrt{\lambda_{1}}\left|\alpha_{1}\right\rangle\left|\beta_{1}\right\rangle+\sin \theta \sqrt{\lambda_{2}}\left|\alpha_{2}\right\rangle\left|\beta_{2}\right\rangle \\
& \sqrt{p_{2}}\left|\psi_{2}\right\rangle=\sin \theta \sqrt{\lambda_{1}}\left|\alpha_{1}\right\rangle\left|\beta_{1}\right\rangle-\cos \theta \sqrt{\lambda_{2}}\left|\alpha_{2}\right\rangle\left|\beta_{2}\right\rangle .
\end{aligned}
$$

\footnotetext{
${ }^{5}$ This will be presented in a forthcomming publication.
} 
Because the ensemble $\mathcal{E}$ is an eigenensemble, we must have either $\left\langle\alpha_{1} \mid \alpha_{2}\right\rangle=0$ or $\left\langle\beta_{1} \mid \beta_{2}\right\rangle=0$ or both. First note that it cannot be the case that both inner products vanish. For otherwise, there will be at least one value of $\theta$ for which $\left|\psi_{1}\right\rangle$ and $\left|\psi_{2}\right\rangle$ will be entangled states. Consequently, let us look at one of the other possibilities: namely, $\left|\alpha_{1}\right\rangle$ and $\left|\alpha_{2}\right\rangle$ are othogonal, but $\left\langle\beta_{1} \mid \beta_{2}\right\rangle \neq 0$. Then we must have $\left|\beta_{1}\right\rangle=e^{i \phi}\left|\beta_{2}\right\rangle$. For otherwise, again - for at least one value of $\theta$ both $\left|\psi_{1}\right\rangle$ and $\left|\psi_{2}\right\rangle$ will be entangled states. The argument goes analogously if $\left|\beta_{1}\right\rangle$ and $\left|\beta_{2}\right\rangle$ are othogonal. That is to say, $\left|\psi_{1}\right\rangle$ and $\left|\psi_{2}\right\rangle$ must indeed be product states but with either identical left factors or identical right factors.

Therefore, just to say it again (but now ignoring phases, since they could have been in the "unitary reshuffling" anyway), we must have either $\left|\alpha_{1}\right\rangle=\left|\alpha_{2}\right\rangle$ or $\left|\beta_{1}\right\rangle=\left|\beta_{2}\right\rangle$. Locking one of these possiblities in, we can go through exactly the same argument for similar "unitary reshufflings" of other pairs of eigenstates. Consistency then gives that we must find either

$$
\rho=\left|\psi_{A}\right\rangle\left\langle\psi_{A}\right| \otimes \rho_{B} \quad \text { or } \quad \rho=\rho_{A} \otimes\left|\psi_{B}\right\rangle\left\langle\psi_{B}\right| .
$$

This completes the proof.

Let us now look at the states with the maximum value 6 for entanglement of assistance. This is the set of all possible mixtures of maximally entangled pure-states. For the simplest non-trivial case of one qubit on either side, this is equivalent to the set of ' $\mathrm{T}$ ' states of Horodecki et al. 114,15] or equivalently the set of real $\square$ density matrices in the magic basis 10$]$.

Next we consider some non-extremal examples. Consider states like $\rho=$ $\operatorname{Diagonal}[\alpha, 0,0,1-\alpha]$ in a product basis. The entropic bound (6) says $A(\rho) \leq$ $H_{2}(\alpha)$ and the diagonal lower bound (20) gives us $A(\rho) \geq H_{2}(\alpha)$ so that $A(\rho)=H_{2}(\alpha)$.

Let us look at a more complicated example, say $\rho=\operatorname{Diagonal}[1 / 3,1 / 3,1 / 3,0]$ in a product basis. The diagonal bound (20) gives us $A(\rho) \geq 2 / 3$. For the fidelity bound, we first calculate $\tilde{\rho}=\operatorname{Diagonal}[0,1 / 3,1 / 3,1 / 3]$ and by the fidelity bound (8) we see that $A(\rho) \leq 2 / 3$. Thus $A(\rho)=2 / 3$. Notice that the entropic bound for this case is just $H_{2}(1 / 3) \approx 0.918$ ebits. Since it does not saturate the entropic bound this state is a good candidate for superadditivity. Let us turn to that next.

\section{Superadditivity}

It has been speculated (and is indicated numerically for a few cases) that the entanglement of formation is additive [16]. This property is very important if it is to be used as a measure of entanglement. Since entanglement of assistance looks dual to entanglement of formation it comes as a bit of a surprise then that the entanglement of formation is superadditive. By this we mean

$$
A\left(\rho_{1}^{\mathrm{AB}} \otimes \rho_{2}^{\mathrm{AB}}\right)>A\left(\rho_{1}^{\mathrm{AB}}\right)+A\left(\rho_{2}^{\mathrm{AB}}\right)
$$

\footnotetext{
${ }_{6}$ The maximum value is $\log _{2}\left(\operatorname{Min}\left[\mathrm{N}_{\mathrm{A}}, \mathrm{N}_{\mathrm{B}}\right]\right)$ ebits. Here, $\mathrm{N}_{\mathrm{A}}$ and $\mathrm{N}_{\mathrm{B}}$ are the dimensions of the Hilbert spaces on Alice's and Bob's side respectively.

${ }^{7}$ upto a phase.
} 
for some density matrices $\rho_{1}^{\mathrm{AB}}$ and $\rho_{2}^{\mathrm{AB}}$. Since the entropic bound is additive, superadditivity is possible only for the states which do not saturate the bound.

The density matrix $\rho=\operatorname{Diagonal}[1 / 3,1 / 3,1 / 3,0]$ which we have just seen in section (3) has an entanglement of assistance $A=2 / 3$. Recall that this state does not saturate the (additive) entropic bound. So it can show superadditivity and in fact it does! If $A$ were additive: $A(\rho \otimes \rho)=2 A(\rho)=4 / 3$. However $\rho \otimes \rho$ can be realized as an ensemble $\mathcal{E}$ which has an average entanglement $E \approx 1.5506$. (This ensemble is shown in detail in appendix A). This being a lower bound on $A(\rho \otimes \rho)$ it shows that entanglement of assistance is superadditive.

Superadditivity of entanglement of assistance means that if Alice, Bob, and Charlie have two copies of the same system then Charlie can give Alice and Bob more entanglement if he makes an entangled measurement on the two copies of the system.

Since entanglement of assistance and entanglement of formation are dual to each other in the sense of replacing a maximization by a minimization, we would expect the superadditivity of the entanglement of assistance to tell us something about the additivity of the entanglement of formation. We have not been able to see a connection as yet but we note here a result [17],

$$
A(\rho)-F(\rho) \leq S(\rho)-\left|S\left(\operatorname{tr}_{\mathrm{A}} \rho\right)-S\left(\operatorname{tr}_{\mathrm{B}} \rho\right)\right|
$$

which connects entanglement of assistance and entanglement of formation. Thus in addition to its own physical significance, entanglement of assistance may turn out to be a useful tool to study the problem of additivity of the entanglement of formation.

\section{Conclusions}

In this paper we have introduced a new measure of entanglement - entanglement of assistance - and studied its properties and found upper and lower bounds for it. We have proved that it is superadditive meaning that, entangled measurements by Charlie give more entanglement to Alice and Bob. We also note here that the superadditivity of entanglement of assistance may throw some light upon the additivity question of the entanglement of formation.

\section{Acknowledgments}

Part of this work was completed during the 1997 Elsag-Bailey - I.S.I. Foundation research meeting on quantum computation. We would like to thank Charles H. Bennet, William K. Wootters and Barbara M. Terhal for illuminating discussions. DPD and JAS would like to thank the Army Research Office for support. CAF was supported by a Lee A. DuBridge Fellowship and by DARPA through the Quantum Information and Computing (QUIC) Institute administered by the US Army Research Office. AT would like to thank The NSF Science and Technology Center for Quantized Electronics Structures, Grant \#DMR 91-20007, for 
support to attend this conference and, IBM Research Division for supporting his summer visit during which this work was initiated. He would also like to thank Prof. David Awschalom for his invaluable support, without which, it would have been impossible to work in this exciting field.

\section{A Superadditivity}

Here we look at the ensemble which gives us an average entanglement more than the additive value of $4 / 3$ for the density matrix discussed is section (4). The ensemble written in the product basis $\left\{\left|00_{\mathrm{A}} 00_{\mathrm{B}}\right\rangle,\left|00_{\mathrm{A}} 01_{\mathrm{B}}\right\rangle, \ldots,\left|11_{\mathrm{A}} 11_{\mathrm{B}}\right\rangle\right\}$ is, $\mathcal{E}=\left\{p_{i},\left|\psi_{i}\right\rangle, i=1, . ., 12\right\}$ where,

$$
\begin{aligned}
\left\{p_{i}\right\} & =\left(\alpha_{1}, \alpha_{1}, \alpha_{1}, \alpha_{1}, \alpha_{1}, \alpha_{1}, \alpha_{2}, \alpha_{2}, \alpha_{2}, \alpha_{2}, \alpha_{2}, \alpha_{2}\right) \\
\left|\psi_{1}\right\rangle & =\left(0,0,0, a, 0,0, b e^{i \pi / 3}, 0,0, b e^{-i \pi / 3}, 0,0, a, 0,0,0\right) \\
\left|\psi_{2}\right\rangle & =\left(0,0,0, a, 0,0,-b e^{i \pi / 3}, 0,0,-b e^{-i \pi / 3}, 0,0, a, 0,0,0\right) \\
\left|\psi_{3}\right\rangle & =\left(0,0,0, a, 0,0, b e^{i 2 \pi / 3}, 0,0, b e^{-i 2 \pi / 3}, 0,0,-a, 0,0,0\right) \\
\left|\psi_{4}\right\rangle & =\left(0,0,0, a, 0,0,-b e^{i 2 \pi / 3}, 0,0,-b e^{-i 2 \pi / 3}, 0,0,-a, 0,0,0\right) \\
\left|\psi_{5}\right\rangle & =(d, 0,0,0,0,0, b, 0,0, b, 0,0,0,0,0,0) \\
\left|\psi_{6}\right\rangle & =(d, 0,0,0,0,0,-b, 0,0,-b, 0,0,0,0,0,0) \\
\left|\psi_{7}\right\rangle & =(0, c, 0,0,0,0, b, 0, c, 0,0,0,0,0,0,0) \\
\left|\psi_{8}\right\rangle & =\left(0, c, 0,0,0,0, b e^{i 2 \pi / 3}, 0, c e^{-i 2 \pi / 3}, 0,0,0,0,0,0,0\right) \\
\left|\psi_{9}\right\rangle & =\left(0, c, 0,0,0,0, b e^{i 4 \pi / 3}, 0, c e^{-i 4 \pi / 3}, 0,0,0,0,0,0,0\right) \\
\left|\psi_{10}\right\rangle & =(0,0, c, 0, c, 0,0,0,0, b, 0,0,0,0,0,0) \\
\left|\psi_{11}\right\rangle & =\left(0,0, c, 0, c e^{i 2 \pi / 3}, 0,0,0,0, b e^{-i 2 \pi / 3}, 0,0,0,0,0,0\right) \\
\left|\psi_{12}\right\rangle & =\left(0,0, c, 0, c e^{i 4 \pi / 3}, 0,0,0,0, b e^{-i 4 \pi / 3}, 0,0,0,0,0,0\right)
\end{aligned}
$$

and,

$$
\begin{aligned}
a & =\left(6-\frac{24}{5+\sqrt{7}}\right)^{-\frac{1}{2}} \approx 0.5912 \\
b & =\left(9-\frac{18}{5+\sqrt{7}}\right)^{-\frac{1}{2}} \approx 0.3879 \\
c & =\left(\frac{5+\sqrt{7}}{18}\right)^{\frac{1}{2}} \approx 0.6517 \\
d & =\left(3-\frac{12}{5+\sqrt{7}}\right)^{-\frac{1}{2}} \approx 0.8361 \\
\alpha_{1} & =\frac{1}{6}-\frac{2}{3(5+\sqrt{7})} \approx 0.0795 \\
\alpha_{2} & =\frac{2}{3(5+\sqrt{7})} \approx 0.0872 .
\end{aligned}
$$


The entanglements for the pure-states forming the ensemble $\mathcal{E}$ are,

$$
\left\{E_{i}\right\}=\left(E_{\alpha}, E_{\alpha}, E_{\alpha}, E_{\alpha}, E_{\beta}, E_{\beta}, E_{\gamma}, E_{\gamma}, E_{\gamma}, E_{\gamma}, E_{\gamma}, E_{\gamma}\right)
$$

where,

$$
\begin{aligned}
& E_{\alpha}=H_{2}\left(a^{2}, a^{2}, b^{2}, b^{2}\right) \approx 1.8824 \text { ebits } \\
& E_{\beta}=H_{2}\left(d^{2}, b^{2}, b^{2}, 0\right) \approx 1.1834 \text { ebits } \\
& E_{\gamma}=H_{2}\left(c^{2}, b^{2}, c^{2}, 0\right) \approx 1.4605 \text { ebits }
\end{aligned}
$$

and average entanglement of this ensemble is,

$$
E(\mathcal{E})=4 \alpha_{1} E_{\alpha}+2 \alpha_{1} E_{\beta}+6 \alpha_{2} E_{\gamma} \approx 1.5506 \text { ebits. }
$$

To find this ensemble we use the following procedure: First, we use a numerical optimization program to find the ensemble which has the maximum average entanglement. Then using the structure of this ensemble, guessing the right phases and using the constraint that the ensemble must give us $\rho \otimes \rho$ we get a quadratic equation which we solve to find the values for $a, b, c, d, \alpha_{1}$ and $\alpha_{2}$.

Note that according to our numerical results, the ensemble presented here achieves the maximum average entanglement consistent with the density matrix. This suggests that this is the entanglement of assistance for it.

\section{References}

1. C.H. Bennett and G. Brassard "Quantum Cryptography: Public Key Distribution and Coin Tossing", Proceedings of IEEE International Conference on Computers Systems and Signal Processing, Bangalore India, December 1984, pp 175-179.; D. Deutsch, A. Ekert, R. Jozsa, C. Macchiavello, S. Popescu, and A. Sanpera, Phys. Rev. Lett. 77, 2818 (1996).

2. C. H. Bennett, G. Brassard, C. Crépeau, R. Jozsa, A. Peres, and W. K. Wootters, Phys. Rev. Lett. 70, 1895 (1993).

3. C. H. Bennett, C. A. Fuchs, and J. A. Smolin, "Entanglement-Enhanced Classical Communication on a Noisy Quantum Channel," in Quantum Communication, Computing and Measurement, edited by O. Hirota, A. S. Holevo, and C. M. Caves (Plenum, New York, 1997).

4. P. W. Shor, Phys. Rev. A 52, 2493 (1995); D. Gottesman, Ph. D. Thesis, California Institute of Technology, 1997, LANL e-print quant-ph/9705052.

5. R. Cleve and H. Buhrman, LANL e-print quant-ph/9704026.

6. J. J. Bollinger, W. M. Itano, D. J. Wineland, and D. J. Heinzen, Phys. Rev. A 54, R4649 (1996).

7. C. H. Bennet, D. P. DiVincenzo, J. A. Smolin, W. K. Wootters, Phys. Rev. A 54, 3824(1996), LANL e-print quant-ph/9604024.

8. C. H. Bennett, H. J. Bernstein, S. Popescu, B. Schumacher, Phys. Rev. A 53, 2046(1996), LANL e-print quant-ph/9511030.

9. L. P. Hughston, R. Jozsa, W. K. Wootters, Phys. Lett. A 183, 14 (1993). 
10. S. Hill, W. K. Wootters, Phys. Rev. Lett. 78 ,5022 (1997), LANL e-print quant-ph/9703041.

11. R. Jozsa, J. Mod. Opt. 41, 2315 (1994).

12. A. Uhlmann, Rep. Math. Phys. 9, 273 (1976).

13. C. A. Fuchs and J. van de Graaf, LANL e-print quant-ph/9712042.

14. R. Horodecki, M. Horodecki, Phys. Rev. A 54, 1838 (1996).

15. R. Horodecki, M. Horodecki, P. Horodecki, Phys. Lett. A 222, 21 (1996).

16. J. A. Smolin, private communication; W. K. Wootters, to appear in Phys. Rev. Lett., LANL e-print quant-ph/9709029.

17. M. Nielsen, private communication; The same result was independently conjectured in a discussion with C. H. Bennett and W. K. Wootters. 